\title{
Oesophageal pouches and diverticula: A pictorial review
}

\author{
N Khan, FCRad (D) \\ F Ismail, FCRad (D), MMed Rad (D) \\ I E A van de Werke, FRCR \\ Department of Radiology, Kalafong Hospital and University of Pretoria
}

Diverticula of the oesophagus are rare. They can occur at any level, and are often defined by their anatomical location. Three categories are recognised, namely pharyngo-oesophageal, parabronchial and epiphrenic. Although these diverticula are often asymptomatic, patients can develop significant problems with dysphagia, regurgitation and aspiration. The causation of oesophageal diverticula is controversial; however, the popular current belief is that most occur because of oesophageal dysmotility. This paper demonstrates the categories of oesophageal diverticula pictorially, including the radiological features and underlying pathology.

S Afr J Surg 2012;50(3):71-75. DOI:10.7196/SAJS.1299

\section{Aetiology}

The classic Rokitansky classification that divides oesophageal diverticula into pulsion and traction diverticula still provides useful information as to the aetiopathogenesis. ${ }^{1}$ The anatomical division according to location into pharyngo-oesophageal (Zenker's), mid-oesophageal (tracheobronchial) and distal (epiphrenic) diverticula is the most suitable for radiological reporting. ${ }^{2,3}$ There are several areas of structural weakness in the wall of the pharynx and oesophagus, where either there are gaps in the muscular walls or the thin strip-like muscles are relatively unsupported by surrounding neck tissue. Transient protrusions through these areas of weaknesses are termed pouches, while persistent protrusions are called diverticula.,

Traction diverticula, as the name implies, are the result of a surrounding inflammatory process with involvement of the oesophagus, such as tuberculous adenitis. Pulsion diverticula, on the other hand, occur as a result of an altered pressure gradient across the oesophageal lumen, which determines the formation of diverticula through the area of least resistance, as is naturally present at Killian's triangle, or due to tearing of the oesophageal wall above a zone of altered motility, e.g. an epiphrenic diverticulum. ${ }^{1}$

\section{Clinical features}

Pharyngeal and oesophageal pouches and diverticula are uncommon before the age of 40; they usually occur in the 7 th and 8th decades of life and are more common in males than females. Typical symptoms include dysphagia, regurgitation of undigested food, borborygmi in the cervical region, chronic coughing, aspiration due to overspill, halitosis and weight loss. ${ }^{6-8}$

\section{Diagnosis}

Most diverticula are asymptomatic and discovered incidentally during investigation of unrelated complaints. When suspected, the investigation of choice is a barium study. It is essential to include the stomach and duodenum in the study to exclude associated conditions such as reflux oesophagitis and hiatal hernia.

\section{Zenker's diverticulum (ZD)}

Pharangeal diverticulum may be posterior, lateral or posterolateral, but the most commonly encountered type is the posterior pulsion diverticulum. Posterior pulsion diverticulum at the cricopharyngeus was first described by Ludlow in 1769. It was later reclassified by and has since been eponymously linked with Zenker. $^{1,4,6}$

$\mathrm{ZD}$ is an acquired pulsion diverticulum through Killian's dehiscence, an area of congenital weakness through the region of the cricopharyngeus muscle (upper oesophageal sphincter; UOS).

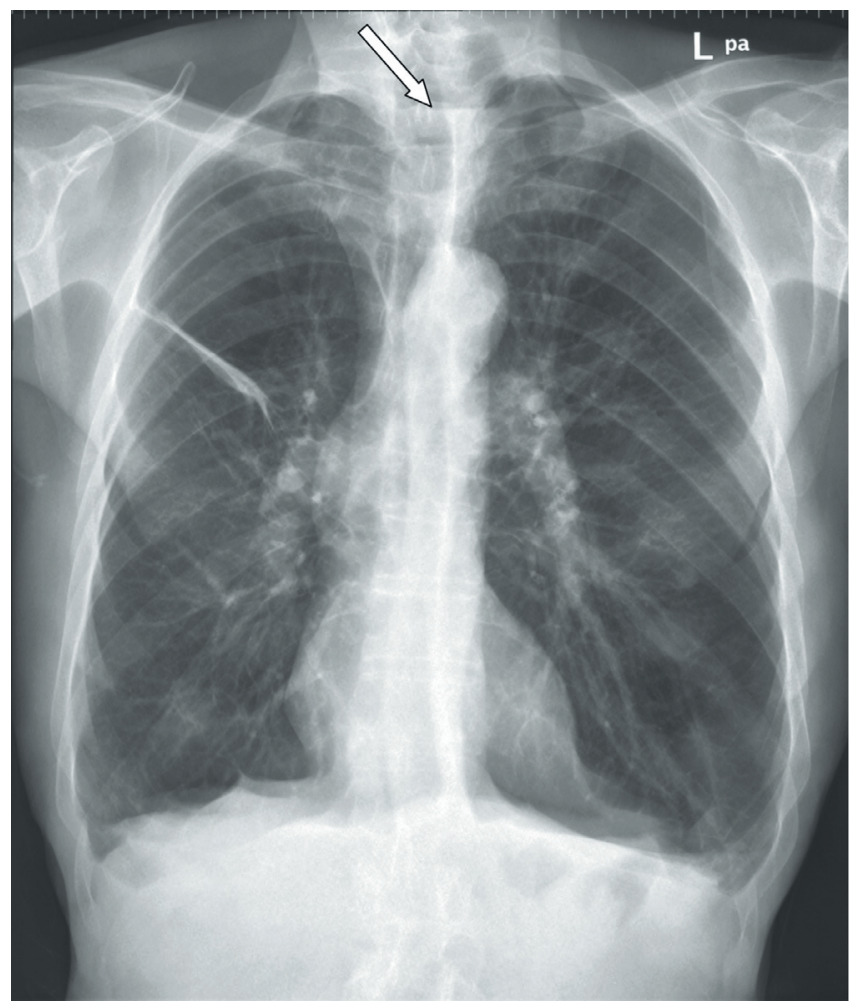

Fig. 1a. Postero-anterior radiograph of the chest, control view for a barium swallow study in a patient with a suspected diverticulum. There is an air/ fluid level in the superior mediastinum (arrow). 


\section{SAJS}

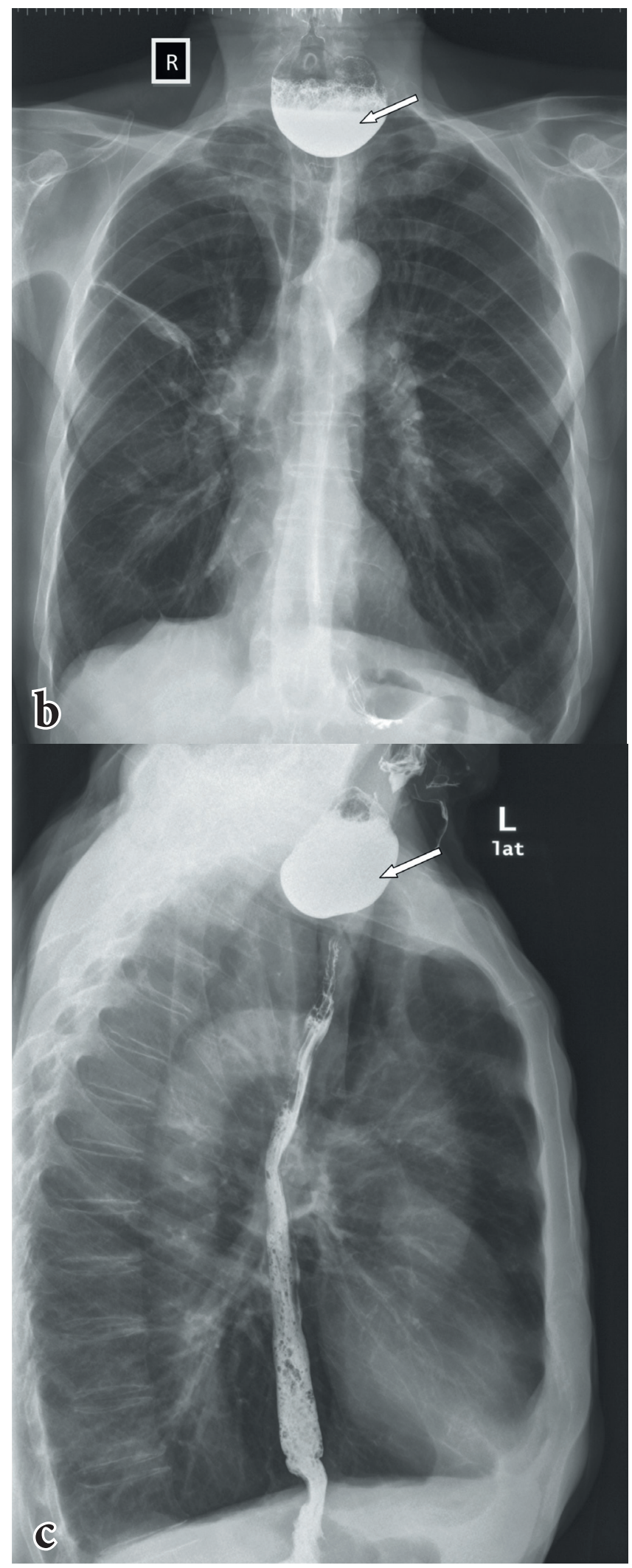

Fig. 1, b and c. Barium swallow study in the same patient shows a large contrast-filled sac in the cervical oesophagus (arrow). The position of the diverticulum is in keeping with a Zenker's diverticulum.

It has been variously described as a protrusion either between the thyropharyngeus and cricopharyngeus portion of the inferior constrictor muscle or between the oblique and horizontal fibres of the cricopharyngeus muscle itself. ${ }^{1,5,69}$ The exact cause of the defect is not known, the prevailing theory for years having been inco-ordination between contraction of the pharynx and relaxation of the upper sphincter, the so-called 'cricopharyngeal achalasia.' On barium study it is seen as a contrast-filled sac posterior to the cervical oesophagus, which may extend into the mediastinum (Fig. 1 , a - c). The diverticulum varies in size from $0.5 \mathrm{~cm}$ to $8 \mathrm{~cm}$. Its opening lies above the cricopharyngeus muscle, which may be prominent or thickened (Fig. 2).

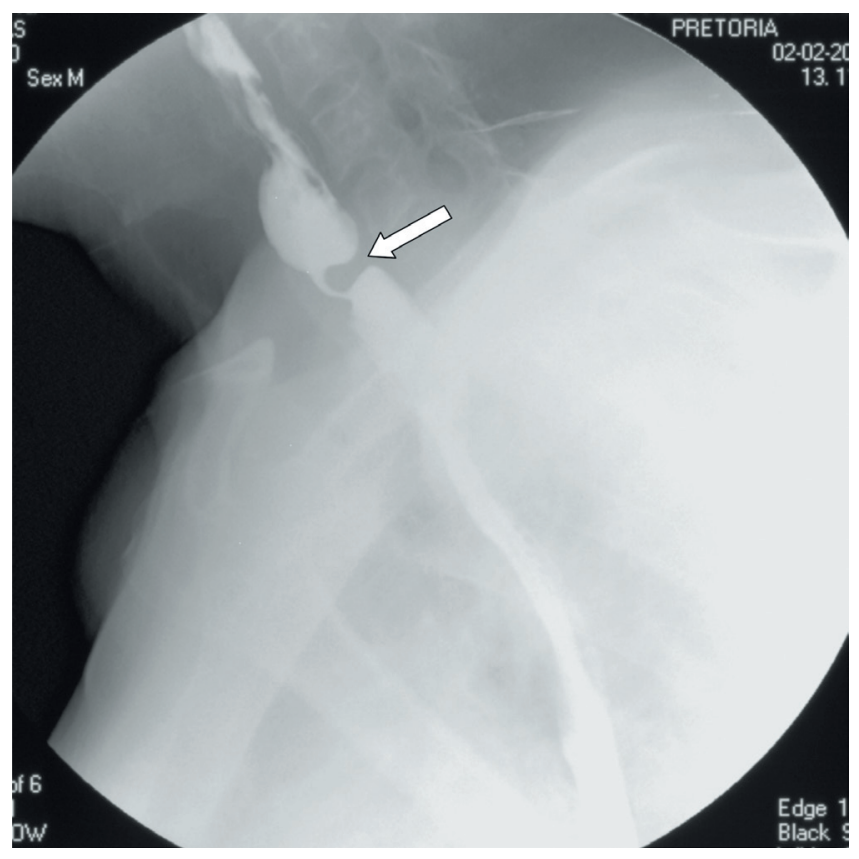

Fig. 2. Oblique view of a barium swallow study demonstrating a prominent and thickened cricopharyngeual impression in the cervical oesophagus (arrow). Note that the mouth of the Zenker's diverticulum is above and posterior, while that of a Killian-Jamieson diverticulum is below and anterior or lateral.

It has recently been postulated that pathophysiology of the pharyngeal pouches involves altered compliance of the cricopharyngeus muscle, detected as impaired pharyngeal opening or raised intrabolus pressure. Histologically it has been observed that in patients with a pharyngeal pouch the cricopharyngeus muscle demonstrates fibro-adipose tissue replacement and fibre degeneration, which it is thought may account for diminished UOS opening. The inter-relationship between gastro-oesophageal reflux and hiatal hernia remains unresolved, though an association has been reported between them. ${ }^{2,6}$

\section{Killian-Jamieson diverticula or pouches (lateral cervical oesophageal pouches)}

The Killian-Jamieson (K-J) diverticulum was first described by Ekberg and Nylander (cited by Chea et al..$^{10}$ ) in 1983. These are transient or persistent protusions through an area of anatomical weakness, the Killian-Jamieson space (not to be confused with Killian's dehiscence), through which the inferior laryngeal nerve passes. This space is bounded superiorly by the cricopharyngeus muscle, anteriorly by the posterior wall of the cricoid cartilage, and inferomedially by the longitudinal tendon of the oesophagus as it 
inserts onto the cricoid cartilage..$^{5,9,10}$ It is commonly asymptomatic, and can be confused clinically with ZD, from which it is easily distinguished radiologically. On barium study (Fig. 3) it lies below the level of cricopharyngeus in the anterolateral wall of the cervical oesophagus, whereas ZD develops at the anatomically weak posterior zone, Killian's dehiscence. ${ }^{10}$ It is $3-20 \mathrm{~mm}$ in size, fills during swallowing and empties after swallowing.
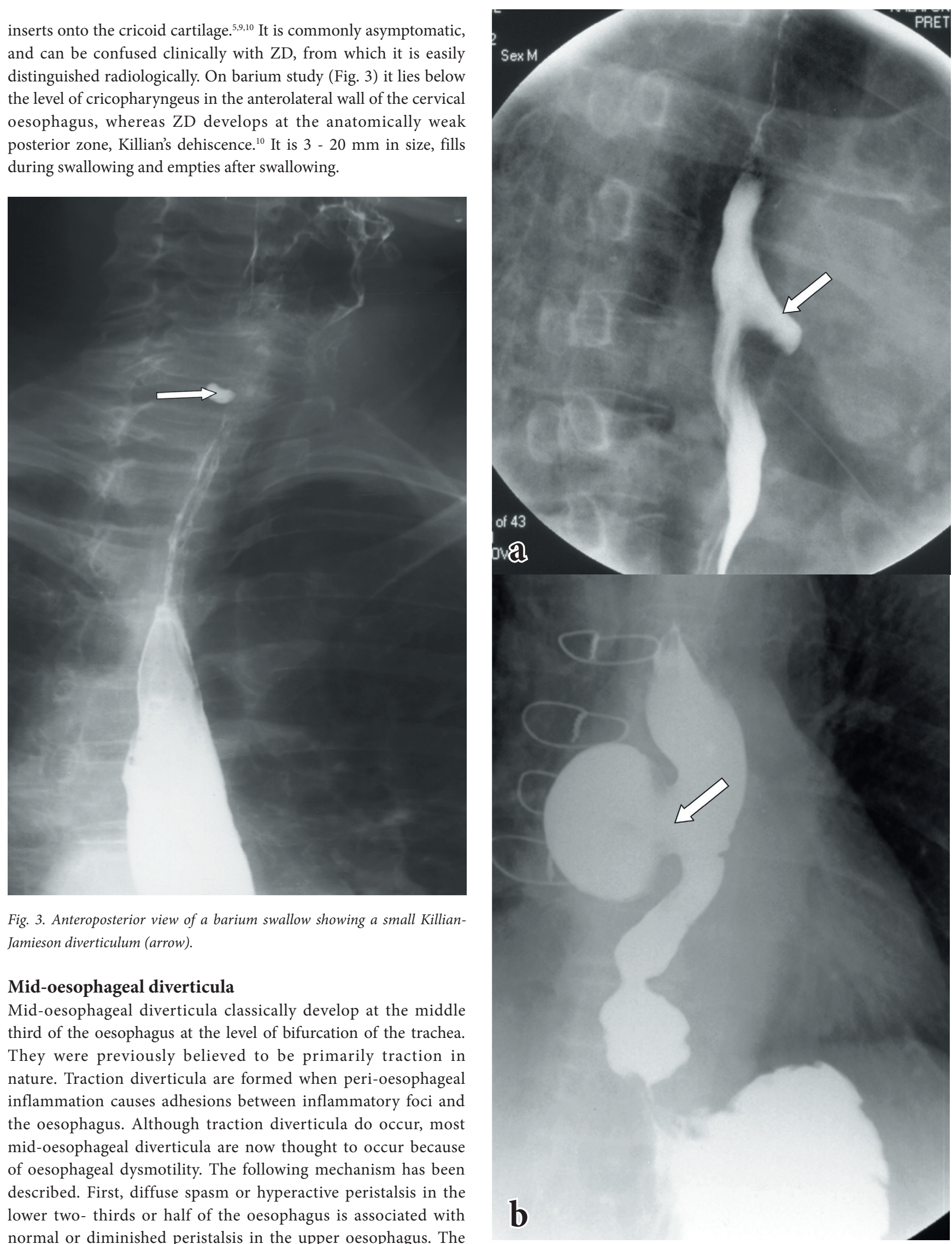

Fig. 3. Anteroposterior view of a barium swallow showing a small KillianJamieson diverticulum (arrow).

\section{Mid-oesophageal diverticula}

Mid-oesophageal diverticula classically develop at the middle third of the oesophagus at the level of bifurcation of the trachea. They were previously believed to be primarily traction in nature. Traction diverticula are formed when peri-oesophageal inflammation causes adhesions between inflammatory foci and the oesophagus. Although traction diverticula do occur, most mid-oesophageal diverticula are now thought to occur because of oesophageal dysmotility. The following mechanism has been described. First, diffuse spasm or hyperactive peristalsis in the lower two- thirds or half of the oesophagus is associated with normal or diminished peristalsis in the upper oesophagus. The persistently increased tone in the distal oesophagus usually produces epiphrenic diverticula. Second, spasm or hyperactive peristalsis in the lower oesophagus is associated with normal or hyperactive peristalsis in the upper oesophagus. The functional

Fig. 4, $a$ and b. Barium swallow study from two patients, both with midoesophageal diverticula. The defect illustrated in $4 a$ is also known as a tracheobronchial diverticulum, pertaining to its location just proximal to the tracheobronchial bifurcation. 


\section{SAJS}

obstruction created when a peristaltic wave encounters the spastic distal oesophagus produces a mid-thoracic diverticulum. Residual congenital attachment between the oesophagus and the tracheobronchial tree in the area of the carina may add an element of traction, accentuate the pulsion abnormality, and facilitate the formation of a mid-thoracic oesophageal diverticula. ${ }^{11}$ Mid-oesophageal diverticula have been associated with several manometric abnormalities, including nutcracker oesophagus, diffuse oesophageal spasm (DOS), achalasia, hypertensive lower oesophageal sphincter and nonspecific oesophageal motility disorder. ${ }^{2,7,8}$ The position and orientation of mid-thoracic diverticula may imply pulsion as a cause. Miskovitz and Steinberg (cited by Rice and Baker ${ }^{11}$ ) reported that pulsion diverticula occur most commonly above the tracheal bifurcation. At this location they project from the left anterolateral border between the aortic arch and the left main bronchus and are known as interaortic-bronchial diverticula. ${ }^{11}$ Classic mid-oesophageal diverticula are small in size with a large neck, and they rarely become symptomatic or require surgical intervention (Fig. 4 , a and b).

\section{Epiphrenic diverticula}

These occur less frequently than ZD, comprising less than $10 \%$ of all oesophageal diverticula. ${ }^{8}$ They were first described by Mondiere in 1833, who attributed them to increased intra luminal pressure. In 1953 Kay reported muscular hypertrophy immediately below

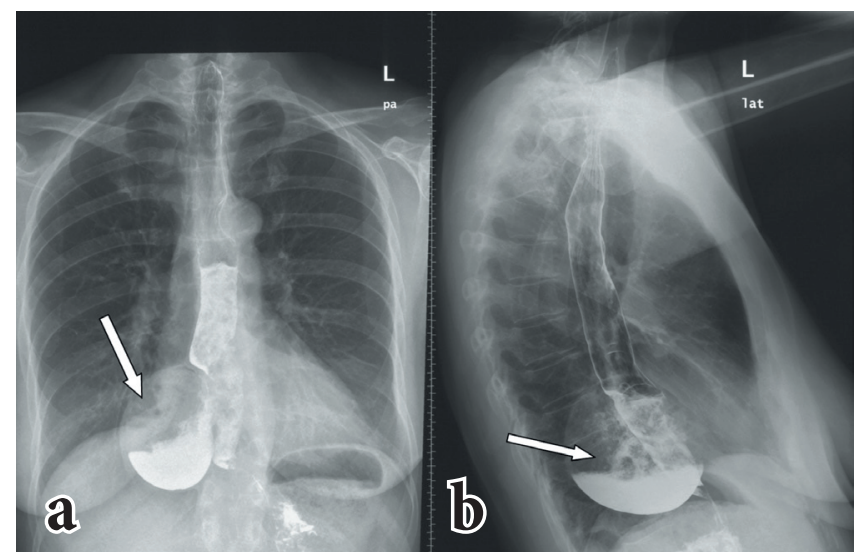

Fig. 5, $a$ and b. Anteroposterior and lateral views of a barium swallow of $a$ patient which demonstrates a large epiphrenic diverticulum (arrow).

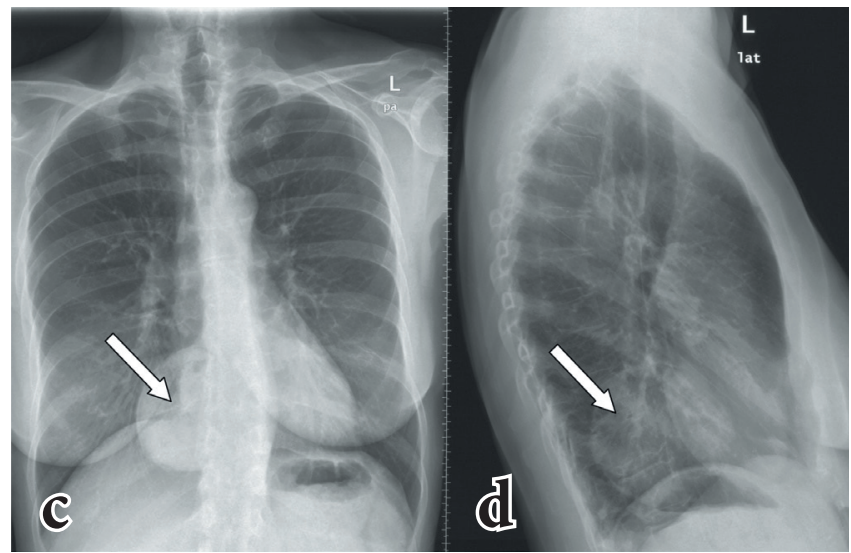

Fig. 5, $c$ and d. Anteroposterior and lateral radiographs of the chest in a patient with a large epiphrenic diverticulum, which mimics a hiatus hernia or a mass lesion (arrow). such diverticula, and later Effer et al. confirmed the consistent hypertrophy of the circular muscle of the terminal oesophagus (cited by Tobin ${ }^{7}$ and Borrie ${ }^{8}$ ). Epiphrenic and mid-oesophageal diverticula have been reported with numerous manometric abnormalities, namely DOS, nutcracker oesophagus, achalasia and cardiospasm. Symptoms result from the cause, and rarely from the diverticulum per se. ${ }^{7,8}$

Epiphrenic diverticula are usually single, but it is not infrequent to find two or more synchronous diverticula. They are best evaluated on barium radiography as large (more than $5 \mathrm{~cm}$ ) diverticula usually $10 \mathrm{~cm}$ from the gastro-oesophageal junction (Fig. 5, a and b). When large, they may appear on plain radiographs as a soft-tissue mass with or without an air/fluid level, mimicking a hiatal hernia (Fig. 5, c and d).

\section{Oesophageal intramural pseudodiverticulosis (OIPD)}

This is an unusual condition in which inflammation leads to dilation of the ducts of the deep mucous glands. OIPD is most likely to be a sequel of chronic oesophagitis, probably reflux. It occurs in elderly patients, usually males, presenting as intermittent or slowly developing dysphagia., ${ }^{3,7}$ On barium swallow examination the ducts appear as tiny $1-5 \mathrm{~mm}$ flask-shaped out-pouchings arranged in clusters of longitudinal rows. Often the neck is not opacified and the diverticulum appears to be separated from the oesophageal lumen by 1 or $2 \mathrm{~mm}$ (Fig. 6). Patients with OIPD and dysphagia respond to dilation of the underlying stricture, while

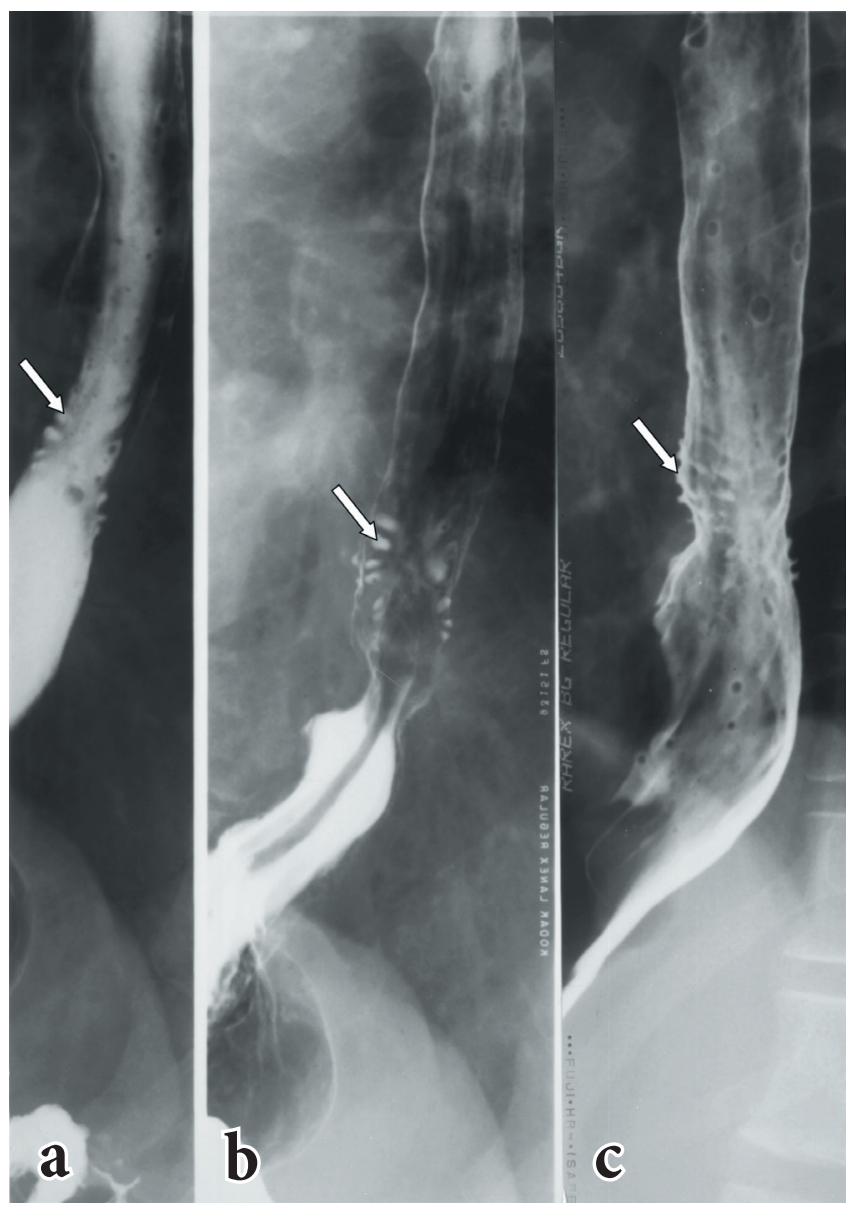

Fig. 6, a - c. A series of images of a barium swallow study demonstrating oesophageal intramural pseudodiverticulosis (OIPD) (arrows). 
those with evidence of Candida infection or gastro-oesophageal reflux should be medically treated.

\section{Conclusion}

Oesophageal diverticula are classified radiologically according to their location as well as their directional orientation. Oesophageal disease such as cricopharyngeal inco-ordination, lower oesophageal spasm, achalasia and sliding hiatal hernia have all been associated with proximal oesophageal diverticula, be they ZD, mid-oesophageal, epiphrenic or indeed intramural pseudodiverticulosis. The previously popular concept of traction was a pleasant teaching tool for surgical concepts, but it is is no longer tenable as there is an established link with disordered oesophageal motility. It is therefore pointless to treat the diverticulum without also correcting the cause of the motor disorder.

Acknowledgement. The authors would like to thank Professor T Mokoena for his valuable contribution.
Disclosure. The authors declare that there are no conflicts of interest pertaining to the subject matter of this article.

\section{REFERENCES}

1. Constantini M, Zaninotto G. Oesophageal diverticula. Best Practice and Research Clinical Gastroenterology 2004;18:3-17.

2. Achkar E. Esophageal diverticula. Gastroenterology and Hepatology 2008;4:691693

3. Gore RM, Levine MS. Textbook of Gastrointestinal Radiology. 3rd ed. Philadelphia: Saunders Elsevier, 2008:475-477.

4. Nelson AR. Congenital true esophageal diverticulum. Ann Surg 1956;145:258-263. 5. Rubesin SE. The pharynx: Structural disorders. Radiol Clin North Am 1994;32:1083-1101

6. Siddiq MA, Sood S, Strachan D. Pharyngeal pouch (Zenker's diverticulum) Postgrad Med J 2001;77:506-511.

7. Tobin RW. Esophageal rings, webs, and diverticula. J Clin Gastroenterol 1998;4:285295.

8. Borrie J, Wilson RLK. Oesophageal diverticula: principal of management and appraisal of classification. Thorax 1980;35:759-767.

9. Perrott J W. Anatomical aspects of hypopharyngeal diverticula. Australian and New Zealand Journal of Surgery 1962;493:304-307.

10. Chea CH, Slow SL, Khor TW, Azim NAN. Killian-Jamieson diverticulum: The rare cervical esophageal diverticulum. Med J Malaysia 2011;66:73-74.

11. Rice TW, Baker ME. Mid-thoracic diverticula. Semin Thorac Cardiovasc Surg 1999;11:352-357. 